\title{
Micrometer per Second
}

National Cancer Institute

\section{Source}

National Cancer Institute. Micrometer per Second. NCI Thesaurus. Code C154858.

A unit of both speed (scalar) and velocity (vector), defined as the distance of one

micrometer travelled per unit time equal to one second. 